\title{
Modelado de volúmenes utilizando análisis geomorfológico para el estudio de sedimentos aportados por deslizamientos en el flanco sur del Volcán Pico de Orizaba, México
}

\section{Gabriel Legorreta-Paulín ${ }^{1, *}$, Rutilio Miguel Castro² y Lilia Arana-Salinas ${ }^{3}$}

\author{
${ }^{1}$ Departamento de Geografía Física, Instituto de Geografía, Universidad Nacional Autónoma de México, \\ Circuito de la Investigación s/n, C.U., Coyoacán, 04510, Ciudad de México, México. \\ ${ }^{2}$ Posgrado en Geografía, Circuito de la Investigación s/ $n$, C.U., \\ Coyoacán, 04510, Ciudad de México, México. \\ ${ }^{3}$ Universidad Autónoma de la Ciudad de México, Av. La Corona 320, Col. Loma la Palma, \\ Gustavo A. Madero, 07160, Ciudad de México, México. \\ *legorretag@hotmail.com
}

\section{RESUMEN}

En la presente investigación se muestra el modelado de la relación entre el área y el volumen de deslizamientos en una cuenca de origen volcánico y sedimentario, utilizando un análisis geomorfológico de relieve para caracterizar la inestabilidad potencial de sus laderas. El uso de este método permite una mejor comprensión de la capacidad del aporte del volumen de material producido por deslizamientos, que depende de cada una de las formas del relieve y a su vez del material que lo compone. El análisis está apoyado en los Sistemas de Información Geográfica (SIG), el uso de drones y de técnicas estadísticas para crear un método integral en la estimación del volumen de deslizamientos. Este método se aplicó a la cuenca del Río Chiquito-Barranca del Muerto en el flanco sur del volcán Pico de Orizaba, México. La cuenca es propensa a los procesos gravitacionales debido a sus condiciones fisiográficas (e.g. depósitos volcánicos y sedimentarios altamente intemperizados que forman terrenos montañosos y escarpados, y que se ven afectados por precipitaciones extremas en la época de lluvias) y antropogénicas (de forestación y cambio de uso de suelo). En el área, se cartografiaron más de seiscientos deslizamientos y se agruparon en distintas formas de relieve. Utilizamos un dron para realizar mediciones a detalle de deslizamientos representativos para establecer una relación empírica entre el área del deslizamiento y su volumen. Esta relación, expresada como una ley de potencias con un exponente de escala, se usó para estimar la contribución potencial del material producido por el total de los deslizamientos en cada forma del relieve en la cuenca. El estudio muestra que el método puede ser útil en lugares remotos con difícil acceso e información topográfica escasa. También muestra que las formas del relieve sedimentarias contribuyen con más sedimentos por kilómetro cuadrado, a pesar de tener menos deslizamientos que las formas volcánicas.

Palabras clave: formas del relieve; SIG; volcán Pico de Orizaba; volúmenes de deslizamientos; vehículos aéreos no tripulados; dron; México.

\begin{abstract}
In this research, the relationship between surface area and volume of landslides in a volcanic watershed is presented, together with the geomorphological analysis of landforms that may lead to slope instability. The use of this method allows a better understanding of the potential landslide volume contribution along homogeneous units. The analysis is supported by Geographic Information Systems (GIS), the use of drones, and statistical techniques to create a comprehensive method for landslide volume estimation for each landform. This approach is applied to the Rio Chiquito-Barranca del Muerto watershed on the south flank of Pico de Orizaba volcano, Mexico. The watershed is prone to gravitational processes because of its physical geographical conditions (highly weathered volcanic and sedimentary deposits that form hilly and steep terrains which are affected by extreme seasonal precipitation) and anthropogenic conditions (deforestation and land use change). In the area, more than six hundred landslides were mapped and grouped into different forms of relief. In the study area, representative landslides were measured in detail with the help of a drone to establish an empirical relationship between the area of the landslide and its volume. This relationship, expressed as a power law with a scale exponent, was used to estimate the potential contribution of the material produced by the landslides in each form of the relief, already pre-established in the basin. The study shows that the method can be useful in remote places with difficult access and sparse topographic information. It also shows that sedimentary landforms contribute with more sediments per square kilometer, even though, they have less landslides than the volcanic landforms.
\end{abstract}

Key words: GIS; landforms; landslide volumen modeling; Pico de Orizaba volcano; unmanned aerial vehicle; drone; Mexico.

\section{INTRODUCCIÓN}

En las zonas montañosas escarpadas, los deslizamientos son uno de los principales procesos naturales que causan pérdidas económicas 
y humanas. Tal es el caso de la provincia fisiográfica del Cinturón Volcánico Trans-Mexicano (CVTM), en la cual son muy comunes los grandes estratovolcanes, domos, conos cineríticos, flujos de lava y campos de ceniza (Lugo-Hubp y Córdoba, 1992; Ferrari et al., 2012). El CVTM es una cadena volcánica del Neógeno que se ubica en la parte Central del país y se extiende $1,000 \mathrm{~km}$ de largo cruzando de este a oeste a la República Mexicana, desde el Océano Pacífico hasta el Golfo de México (Lugo-Hubp y Córdoba, 1992; Ferrari et al., 2012). Los relieves volcánicos en esta provincia tienen un gran potencial para producir deslizamientos debido a que se encuentran en una zona tectónica activa con alta tasa de sismos, fracturas y fallas, vulcanismo activo y lluvias intensas, además de fuertes pendientes cubiertas con depósitos piroclásticos intemperizados, pobremente consolidados y una cobertura vegetal escasa debido a la tala inmoderada (CENAPRED, 2017). Estos deslizamientos incrementan la disponibilidad de sedimentos que promueven la formación de lahares de gran magnitud a lo largo de los cauces de los ríos (Lugo-Hubp et al., 2005; Capra et al., 2018; Díaz et al., 2019). La zona de estudio se ubica en la parte oriental del CVTM, donde se encuentra el volcán Pico de Orizaba (5,675 m s.n.m.), el estratovolcán más alto de México. En específico, la presente investigación se concentra en la cuenca del Río Chiquito-Barranca del Muerto, ubicada en el flanco sur del volcán (Figura 1), para evaluar el volumen potencial de sedimentos aportado por los deslizamientos utilizando un vehículo aéreo no tripulado (VANT), métodos estadísticos y Sistemas de Información Geográfica (SIG). La cuenca está rodeada por una zona montañosa con una alta dinámica de erosión en rocas volcánicas y sedimentarias. El área es naturalmente propensa a deslizamientos debido a la interacción de factores geomorfológicos (pendientes pronunciadas, alta energía del relieve y gran erosión vertical), geológicos (sismicidad, rocas y depósitos volcánicos, y sedimentarios muy intemperizados) y antrópicos (agricultura de subsistencia y deforestación) (Rodríguez et al., 2006; Rodríguez et al., 2011; AlanisAnaya, 2017). Como resultado de los sedimentos removilizados por los deslizamientos hacia los cauces de los ríos, se producen flujos de escombros episódicos durante la época de lluvias. Por ejemplo, en 2003 y 2011, las precipitaciones provocaron deslaves y flujos de escombros que ocasionaron daños financieros y pérdidas de vidas en poblaciones ubicadas en las áreas bajas y planas de la cuenca (CFE, 2006; Rodríguez et al., 2011; Legorreta-Paulín et al., 2014). El riesgo asociado con los deslizamientos que constantemente ocurren en el sistema fluvial, con la acumulación de rocas, suelo y escombros, aumenta por la expansión de los asentamientos humanos y la actividad económica en la cuenca. Esto crea una situación potencialmente peligrosa para los habitantes de ciudades como Córdoba, Orizaba, Río Blanco, Nogales y Ciudad Mendoza, asentada en la parte baja de la cuenca. A pesar de la importancia del volumen de materiales aportado por los deslizamientos, hay una investigación limitada sobre el tema. Para abordar los procesos de producción de sedimentos por deslizamientos en el área de estudio, esta investigación utilizó 15 formas del relieve previamente definidas en otro estudio (Alanis-Anaya, 2017). La estimación del volumen de materiales aportados por los deslizamientos se obtuvo a través de una relación empírica con base en los valores geométricos (área y volumen) de los deslizamientos representativos medidos durante el trabajo de campo utilizando un VANT. Con los resultados aquí obtenidos, se da un marco de referencia viable para modelar el volumen y la distribución de sedimentos aportados por deslizamientos en grandes áreas carentes de información espacial detallada. El método permite, en primer lugar, la eliminación de la mayoría del ruido (árboles, arbustos, etc.) del modelo digital de superficie derivado del VANT para la definición de la topografía previa y actual de los deslizamientos y, en segundo lugar, la obtención de morfometría con la cual se deriva la ley de potencias para calcular el volumen por formas del relieve.

\section{ANTECEDENTES}

El volumen de material aportado por los deslizamientos a la red fluvial puede ser muy variable y difícil de calcular en áreas de gran extensión y complejidad geológica, geomorfológica y de uso de suelo (Broeckx et al., 2016). A nivel mundial, se emplean las formas del relieve que representan áreas homogéneas para comprender las condiciones que controlan la distribución, frecuencia y tamaño de los deslizamientos, así como el volumen desplazado (Iwahashi et al., 2001; Guzzetti et al., 2005; Guzzetti et al., 2009; Broeckx et al., 2016). Para estimar los

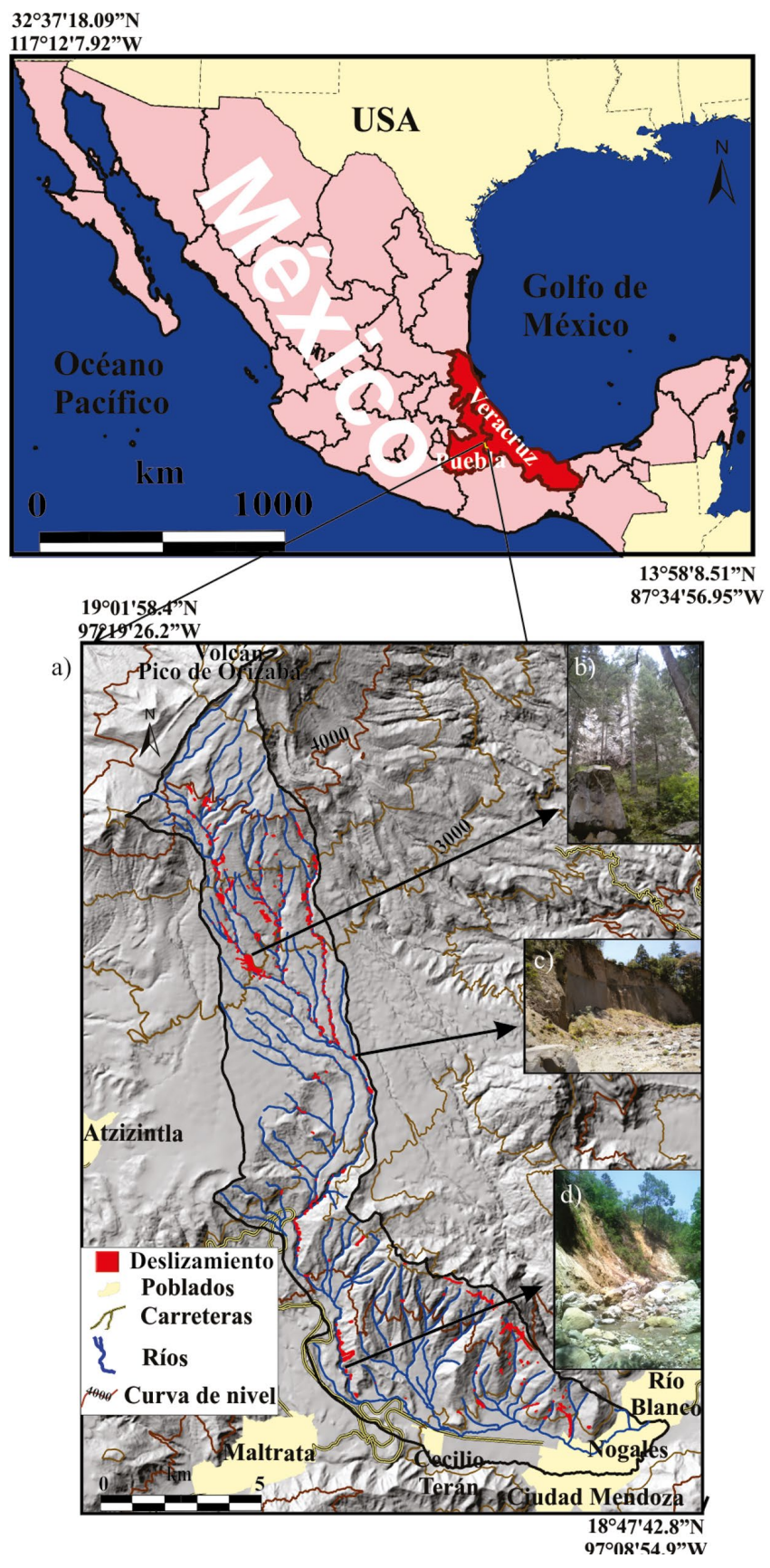

Figura 1. Área de estudio, cuenca del Río Chiquito-Barranca del Muerto. a) Inventario de deslizamientos; b) caída de rocas a lo largo de un frente de lava; c) deslizamiento de escombros en terrenos volcánicos; d) deslizamiento de escombros en terrenos sedimentarios. 
volúmenes de materiales aportados por los deslizamientos, los estudios a escala regional utilizan los inventarios de deslizamientos y la geometría de estos mismos para establecer una ley de potencias que vincula el área de deslizamientos (A) y su volumen (V) (Kaldaron-Asael et al., 2008; Guzzetti et al., 2009; Wenkey et al., 2012). En tales trabajos se utilizan diferentes técnicas para obtener las características morfográficas y morfométricas de los deslizamientos. Algunas de estas técnicas utilizan la detección remota a través de satélites y vuelos aéreos que utilizan sensores multiespectrales, LiDAR, radares de apertura sintética (SAR) y cámaras digitales (Francioni et al., 2018). En los últimos años, los VANT se utilizan cada vez más para recopilar imágenes aéreas de alta resolución y generar cartografía geomorfológica, modelos digitales del terreno y mediciones morfográficas y morfométricas (Colomina y Molina, 2014; Edmonds, 2017). En México, se han realizado esfuerzos para evaluar y cartografiar la susceptibilidad de deslizamientos (Oliva-González et al., 2014; Hernández y Ruiz 2016). Sin embargo, las investigaciones aisladas rara vez abordan el modelado y cálculo de volumen de materiales aportados por los deslizamientos a través de VANT, SIG, y enfoques heurísticos y probabilísticos (Muñoz-Salinas et al., 2009; Ruiz et al., 2017; CENAPRED, 2017). En el área de estudio, 12 formas de relieve se definieron mediante el uso de reglas genéricas de gradiente y forma de laderas, tipo de roca y densidad de deslizamientos adoptadas por el Departamento de Recursos Naturales del Estado de Washington, DNR, División de Prácticas Forestales (WS-DNR:FP) 2006 (WS-DNR:FPD, 2006; Legorreta-Paulín et al., 2014).

Utilizando criterios heurísticos-geomorfológicos y adaptando el protocolo de WS-DNR:FP se establecieron siete formas volcánicas del terreno para una subcuenca de $5.2 \mathrm{~km}^{2}$ (subcuenca el Estado) dentro del área de estudio (Legorreta-Paulín et al., 2017). El volumen total aportado por 121 deslizamientos fue calculado en $\sim 296373 \mathrm{~m}^{3}$ estableciendo una ley de potencias $\mathrm{V}=0.3235192 * \mathrm{~A}^{1.4095}$ mediante mediciones hechas con cinta, estadal y GPS diferencial en 19 deslizamientos (Legorreta-Paulín et al., 2017).

La cuenca del Río Chiquito-Barranca del Muerto fue reclasificada en 15 formas del relieve volcánicas y sedimentarias derivadas de la interpretación de fotografías aéreas, uso de suelo, el trabajo de campo geológico, criterios geomorfológicos y parámetros morfométricos (hipsometría, densidad de drenaje, erosión vertical y pendiente) (Alanis-Anaya, 2017). Estas formas del relieve fueron establecidas con el objetivo de obtener un mapa de áreas susceptibles a inestabilidad de ladera por cambio de uso de suelo utilizando análisis multicriterio. El trabajo calcula un índice de erosividad para las formas del relieve (Alanis-Anaya, 2017), pero no establece el volumen potencial de material removilizado por los deslizamientos.

\section{ÁREA DE ESTUDIO}

La cuenca del Río Chiquito-Barranca del Muerto tiene un área de $\sim 105 \mathrm{~km}^{2}$, y se ubica entre los estados de Puebla y Veracruz, en el flanco sur del volcán Pico de Orizaba (Figura 1). Desde el punto de vista administrativo, la cuenca pertenece a los municipios de Chalchicomula de Sesma y Atzitzintla en el estado de Puebla, y a los municipios de Camerino Z. Mendoza, La Perla, Maltrata, Mariano Escobedo y Nogales del estado de Veracruz. Su punto más alto es 5675 m s.n.m. y el punto más bajo de la cuenca es de $1280 \mathrm{~m}$ s.n.m. Las pendientes generalmente varían de $\sim 5^{\circ}$ en áreas planas a $\sim 65^{\circ}$ en las laderas de pendientes escarpadas. Las rocas volcánicas de composición andesítica y dacítica comprenden casi el $70 \%$ de la litología de la cuenca y el resto está cubierto por rocas sedimentarias (calizas, dolomitas y lutitas del Cretácico). Por encima de los 3000 m s.n.m., el clima se caracteriza como semi-frío subtropical y templado subtropical, y por debajo de esta elevación el clima es clasificado tipo subhúmedo (García, 2004). Las precipitaciones se producen principalmente de mayo a noviembre y de febrero a abril, con valores anuales que oscilan entre los $\sim 1100 \mathrm{~mm}$ y 927 mm. (Jauregui, 1995; García, 2004). Las actividades humanas y la deforestación han conducido a acelerar los procesos erosivos y gravitacionales en el área. Por ejemplo, de 2003 a 2012, $2.64 \mathrm{~km}^{2}$ de bosque fueron reemplazados por áreas de pastos, agricultura y asentamientos humanos (Alanis-Anaya, 2017).

\section{MÉTODOLOGÍA}

En un estudio previo se compiló un mapa detallado de deslizamientos que dio lugar a un inventario de la zona de estudio (Legorreta-Paulín et al., 2014). Este inventario se realizó a través de trabajo de campo y la interpretación de dos conjuntos de fotografías aéreas tomadas en los años de 1994 y 2008 a escalas de 1:20000 a 1:10000 respectivamente. El inventario clasifica los procesos de remoción en masa en deslizamientos superficiales como: flujos de escombros, deslizamientos de escombros y deslizamientos de asentamiento profundo como: flujos de tierra y caídas de rocas mediante el uso del Protocolo de Zonación de Riesgos de Deslizamientos del Departamento de Recursos Naturales del Estado de Washington, USA (WS-DNR:FPD, 2006). Este mapa se actualizó para el período del año 2003 a diciembre del año 2017 a través de trabajo de campo e imágenes satelitales SPOT pancromáticas. Siguiendo este procedimiento, se reconocieron 165 nuevos deslizamientos activos, para un total de 607 procesos de remoción en masa para el área de estudio (Figura 1). La cabecera, la zona de evacuación y el depósito de los deslizamientos se digitalizaron por separado como polígono en ArcGIS ${ }^{\circledast}$. Así mismo, se colectaron algunos atributos de los deslizamientos, los cuales incluyen: área, perímetro, longitud, ancho, tipo de deslizamiento, actividad actual, posición en la ladera, porcentaje de cubierta vegetal, forma de relieve, pendiente y uso de suelo. Todos estos atributos se vincularon con la información espacial de cada deslizamiento y se almacenaron en ArcGIS .

Las áreas que aportan más volúmenes de material en la cuenca se definieron con base en las formas de relieve previamente determinadas por Alanis-Anaya, 2017 (Figura 2): Relicto de colapso volcánico, coladas históricas de lava (años 1545, 1566 y 1613), coladas de lava (Pleistoceno superior), coladas de lava de Sierra Negra (Pleistoceno), laderas cubiertas de depósitos piroclásticos, rampa piroclástica, rampa piroclástica indiferenciada, mesa volcánica, lomeríos basálticos, montañas plegadas (calizas-esquistos del Cretácico), lomeríos plegados (caliza-dolomita del Cretácico), piedemonte entre 3-6 ${ }^{\circ}$, piedemonte menor a $3^{\circ}$, rampa fluvioglacial y valles fluviales (Alanis-Anaya, 2017). Para el análisis del volumen de deslizamientos, los valles fluviales no se tomaron en cuenta, ya que estos se incluyen en las otras formas de relieve. Sin embargo, el porcentaje de deslizamientos y el volumen que afecta a cada unidad de relieve a lo largo de los ríos se da en la sección de Resultados.

Para obtener los volúmenes de sedimentos aportados por los deslizamientos, se estableció una relación empírica de área-volumen en forma de ley de potencias. Del inventario de deslizamientos, aproximadamente el 10\% (61 deslizamientos) se midieron en el campo. No se hizo ninguna distinción entre deslizamientos superficiales y deslizamientos de asentamiento profundo. El área y el volumen de las cabeceras de los deslizamientos se midieron a partir de las imágenes adquiridas con un VANT (Phantom 4 Pro) con una cámara con sensor CMOS de 1" y 20 mega píxeles de resolución. Se utilizó el programa de captura Pix $4 \mathrm{D}^{\bullet}$ para la planeación de captura de vuelo. Se usó un plan de vuelo de doble malla con una superposición entre cada foto aérea del $80 \%$ (Figura 3 ). 


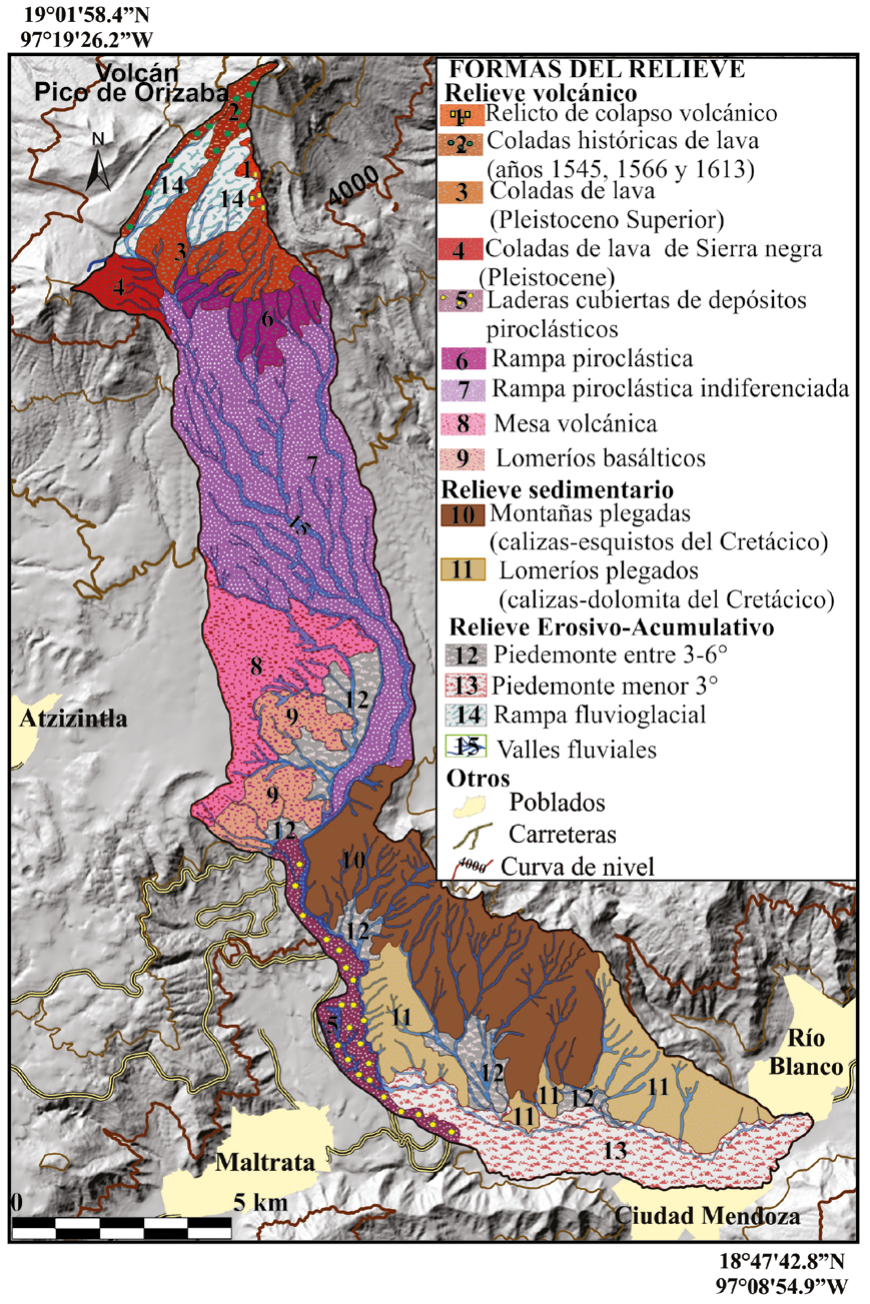

Figura 2. Formas del relieve (Fuente: Modificado de Alanis-Anaya, 2017).

Las fotos aéreas se adquirieron con una altura de vuelo promedio de $100 \mathrm{~m}$, y a una velocidad de $1.5 \mathrm{~m} / \mathrm{s}$. A la par, en campo se tomaron puntos de control terrestre con GPS diferencial para georreferenciar las fotografías. El pos-procesamiento de las imágenes obtenidas se realizó utilizando un procedimiento estándar del software ArgisoftPhotoScan ${ }^{\circledR}$ modificado por Ouédraogo et al. (2014). Este procedimiento crea una nube de puntos densa con valores de $X, Y$ y $Z$. La nube de puntos densa se procesó mediante el uso del programa dem_lidar_generation (Parrot, 2011) para generar un modelo digital de superficie (MDS) estándar de alta resolución. Se estableció una resolución de $50 \mathrm{~cm}$ por píxel. Este modelo incluye no sólo la topografía del terreno, sino también otros elementos naturales y antrópicos (Figura 4a).

En este estudio, dichos elementos los consideramos como ruido que afecta la definición de la topografía. Para eliminar el ruido y adquirir un Modelo Digital de Terreno (MDT) que represente sólo la topografía, se procesó el MDS. El proceso incluyó utilizar un MDT derivado de curvas altimétricas con una equidistancia de $10 \mathrm{~m}$ de un mapa topográfico a escala 1:20000 (INEGI, 2015) (referido aquí como MDT de baja resolución), un Modelo de Altura del Dosel (MAD), histogramas altimétricos, interpolaciones, ponderaciones y un filtro de pasa baja para obtener un MDT de $50 \mathrm{~cm}$ (denominado aquí MDT de resolución moderada). A cada curva del MDT de baja resolución se les extrajo los valores de $X, Y$ y $Z$ de sus nodos. Estos valores se usaron para realizar una interpolación lineal multidireccional (Parrot, 2012;
Parrot, 2016; Šiljeg et al., 2019). Con lo anterior se generó un MDT de $50 \mathrm{~cm}$ de resolución para cada uno de los 61 deslizamientos medidos, mismo que se usó en las etapas posteriores (Figura 4b-4d).

El MAD, que representa las diferencias de altura entre el suelo y la parte superior de los árboles se creó con un tamaño de píxel de $50 \mathrm{~cm}$ utilizando el software SelectSlice (Parrot, 2018a) (Figura 5a). El software resta el MDT de baja resolución (derivado de las curvas topográficas) al MDS de alta resolución (derivado del VANT). La distribución de las diferencias de altura en el MAD se evaluó con un histograma y se ponderó de manera heurística para eliminar el ruido con el programa Shaving (Parrot, 2018b). Por ejemplo, si existiera en el MAD un píxel con una diferencia de altura de $10 \mathrm{~m}$, y se pondera un 5\%, el programa Shaving restará $9.6 \mathrm{~m}$ en lugar de $10 \mathrm{~m}$ al MDS. El MDT resultante todavía presentará algunas irregularidades topográficas. Para ello se aplicó un filtro pasa baja mediante el cual se eliminan parcialmente las irregularidades restantes y con ello se crea el MDT final de resolución moderada. Este MDT de resolución moderada representa la superficie actual que ha sido afectada por un deslizamiento (Figura 5c). Al utilizar este procedimiento, se estimó un error máximo vertical para el área de estudio entre 0 y $1 \mathrm{~m}$.

En ArcMap ${ }^{\oplus}$, la superficie de deslizamiento actual (representada por el MDT de moderada resolución) se utilizó para crear la superficie previa al deslizamiento. La superficie previa se obtuvo de manera heurística, a través de observación de campo y mediante una interpolación. La observación en campo permitió también validar que no existieran relictos intactos de topografía dentro de los deslizamientos. De haber existido, hubieran sido tomados en cuenta para el proceso de interpolación. Con cada deslizamiento verificado, se derivaron las curvas altimétricas con una equidistancia de $50 \mathrm{~cm}$ del MDT de moderada resolución. Una vez creadas, se eliminaron las curvas dentro del área

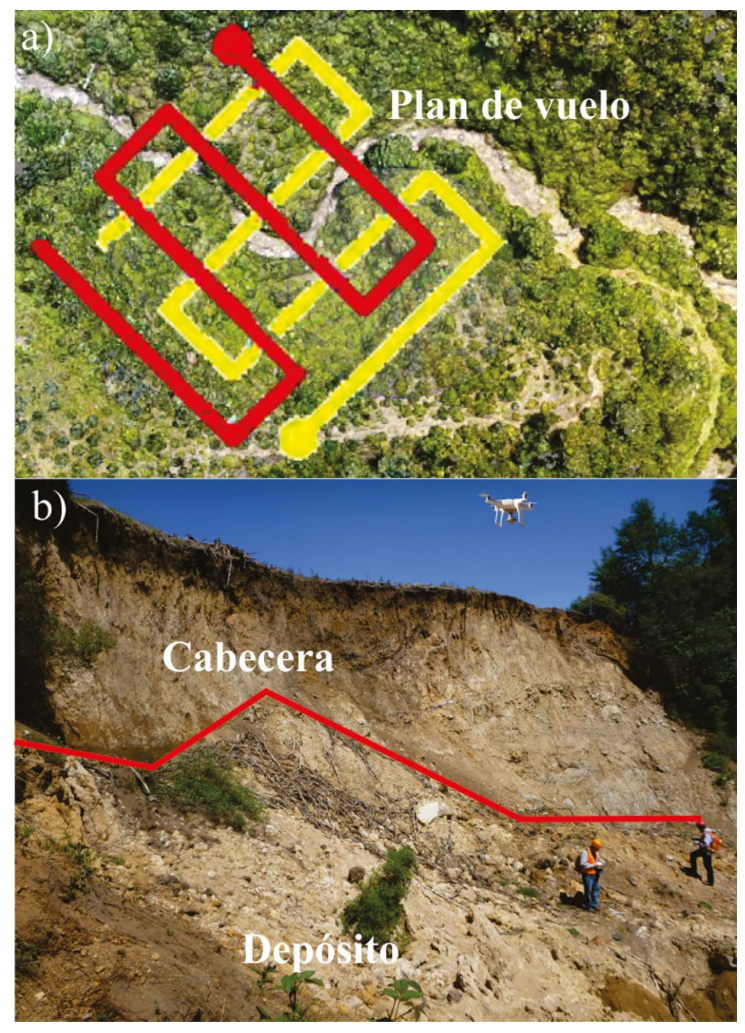

Figura 3. a) Plan de vuelo de un deslizamiento; b) mediciones con VANT sobre la cabecera y el depósito de un deslizamiento de asentamiento profundo. 

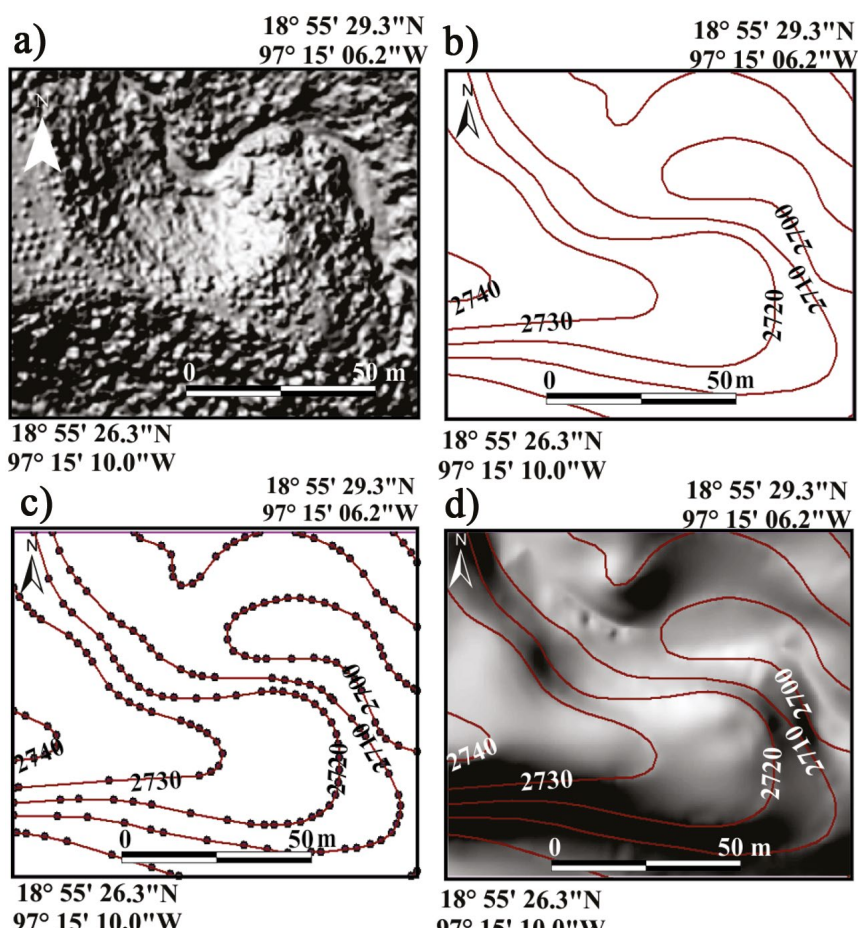

$18^{\circ} 55^{\prime} 26.3^{\prime \prime} \mathrm{N}$ $97^{\circ} 15^{\prime} 10.0 " \mathrm{~W}$

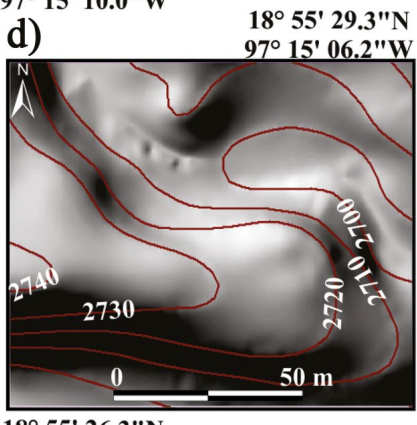

$18^{\circ} 55^{\prime} 26.3$ " N

$97^{\circ} 15^{\prime} 10.0 " \mathrm{~W}$

Figura 4. Obtención del MDS y del MDT de baja resolución. a)MDS de un deslizamiento de asentamiento profundo adquirido a partir de las imágenes obtenidas con el VANT. b) Curvas de nivel con intervalos de $10 \mathrm{~m}$ usadas para la obtención del MDT de baja resolución, c) extracción de los valores $X, Y$ y $Z$ de los nodos de las curvas de nivel para usarlos en la interpolación multidireccional; d) MDT de baja resolución resultante de la interpolación.

del deslizamiento mediante la herramienta erase de ArcMap ${ }^{\circ}$. A las curvas remanentes se les extrajo los valores de $X, Y$ y $Z$ de sus nodos para realizar la interpolación lineal multidireccional (Parrot 2012; Parrot, 2016; Šiljeg et al., 2019) con la que se obtuvo la superficie previa al deslizamiento. El volumen de 61 deslizamientos se calculó a partir de la diferencia algebraica entre el MDT de resolución moderada y la superficie previa al deslizamiento, multiplicado por el área del píxel. Se utilizó un diagrama de dispersión para establecer el grado de relación entre el área y el volumen de los deslizamientos. El diagrama mostró que el $71 \%\left(R^{2}\right)$ del volumen es explicado por el área de los deslizamientos. Al usar el área y el volumen de los deslizamientos medidos en campo, se calculó una ley de potencias con valor a $\mathrm{V}=0.70242506{ }^{*} \mathrm{~A}^{1.1263}$. Esta fórmula se usó en el SIG para calcular el volumen de los 546 deslizamientos no seleccionados para las mediciones de campo (Figura 6).

\section{RESULTADOS}

El volumen total de deslizamientos se calculó para cada forma de relieve (excluyendo la forma de relieve llamada valles fluviales) y para toda la cuenca, el volumen se clasificó en tres intervalos: bajo, menor a $15000 \mathrm{~m}^{3}$, medio, de $15000 \mathrm{~m}^{3}$ a $30000 \mathrm{~m}^{3}$ y alto, mayor a $30000 \mathrm{~m}^{3}$ (Tablas 1, 2 y Figura 7). Los resultados muestran que los deslizamientos tienen una contribución potencial de $\sim 1547657 \mathrm{~m}^{3}$ de material hacia la cuenca. Entre las formas del relieve, una unidad volcánica y dos formas del relieve sedimentarias contribuyen con la mayor cantidad de sedimentos por kilómetro cuadrado. La rampa piroclástica indiferenciada es la forma de relieve más grande en la cuenca, cubre el $25 \%$, seguida de las montañas plegadas (calizas-esquistos del Cretácico) con
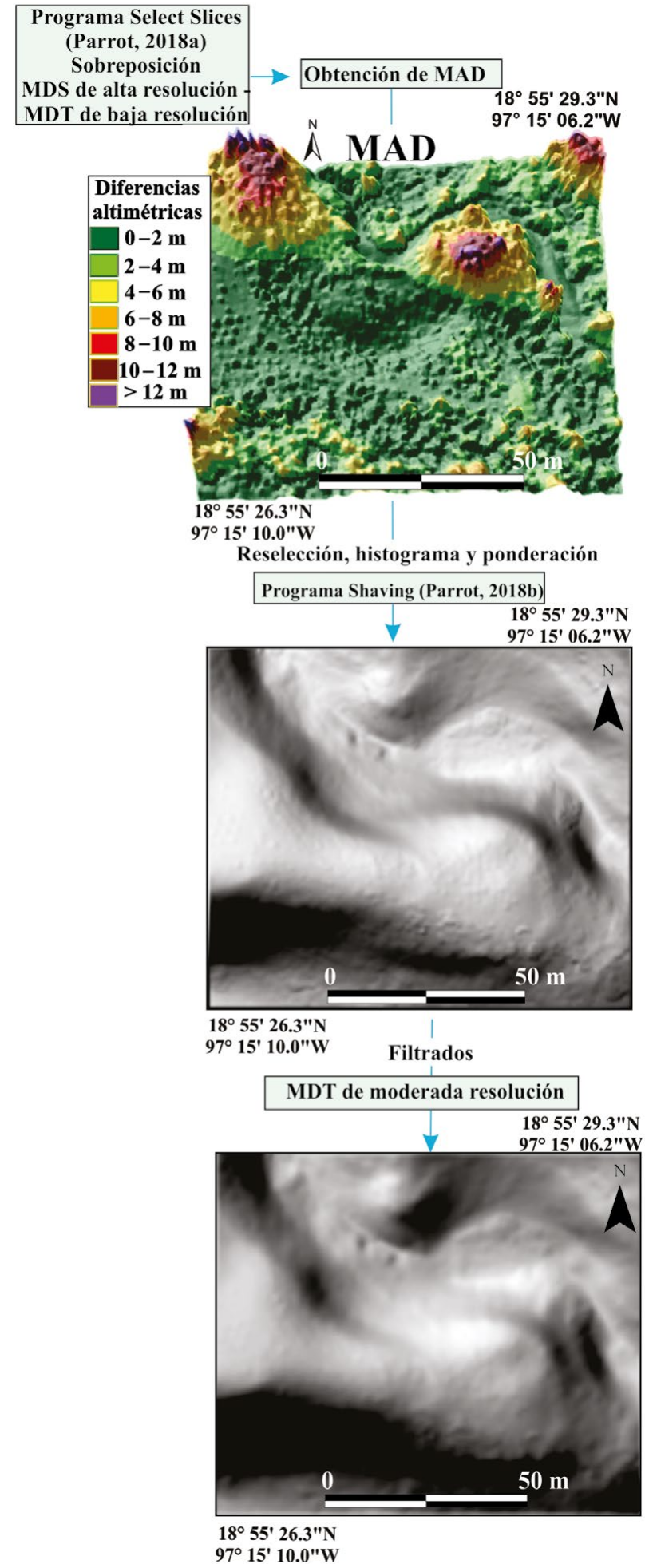

Figura 5. Procedimiento para obtener el MDT de moderada resolución.

$20 \%$ y la unidad de laderas plegadas (calizas-dolomitas del Cretácico) con $11 \%$. La unidad volcánica está formada por depósitos de flujos piroclásticos, de escoria y de pómez que cubren todos los flancos del volcán. Esta forma de relieve incluye 356 (59\%) de los deslizamientos cartografiados, los cuales contribuyen con $829718 \mathrm{~m}^{3}$ (54\%) de los sedimentos. De sus 356 deslizamientos, 266 se encuentran a lo largo 


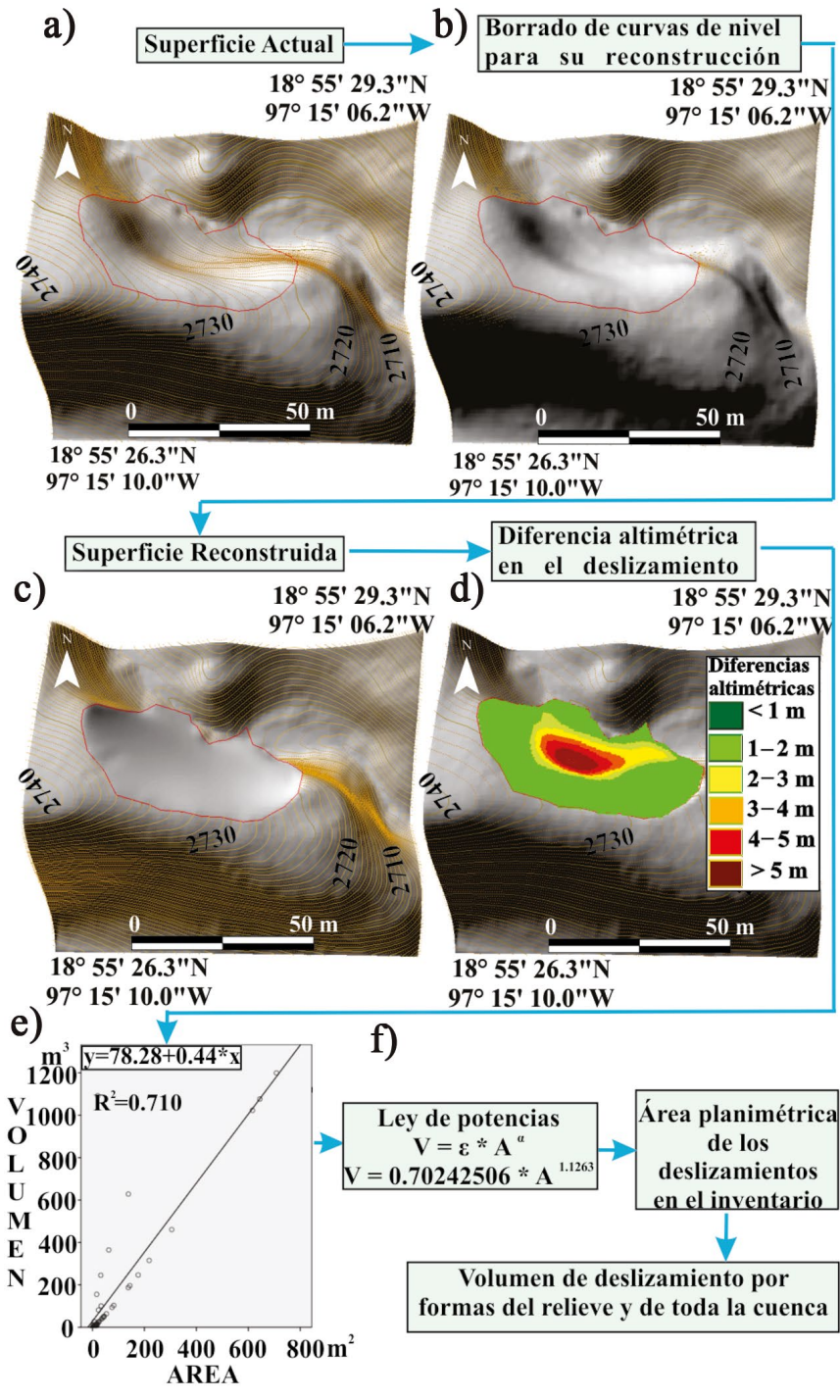

Figura 6. Procedimiento para el cálculo de volúmenes de las formas del relieve y de la cuenca. a) curvas de nivel con la topografía actual del deslizamiento; b) eliminación de las curvas de nivel dentro del límite del deslizamiento; c) interpolación y filtrado para crear la superficie previa al deslizamiento; d) volumen del deslizamiento; e) grafica de dispersión para obtener el grado de relación entre área y volumen de los deslizamientos muestreados; $\mathrm{f}$ ) ley de potencias usada para calcular el volumen total por formas del relieve y de toda la cuenca.

del sistema de arroyos que afectan el $18 \%$ de su área. Las montañas plegadas (calizas-esquistos del Cretácico) se encuentran en el suroeste de la cuenca e incluye 111 (18\%) de los deslizamientos cartografiados. Estos contribuyen con $188115 \mathrm{~m}^{3}$ (12\%) de los sedimentos. De sus 111 deslizamientos, 41 están a lo largo del sistema de arroyos que afectan el $13 \%$ de su área. La unidad de las laderas plegadas (calizas-dolomitas del Cretácico) se encuentra en el sureste de la cuenca e incluye 91 (15\%) de los deslizamientos. Estos aportan $420508 \mathrm{~m}^{3}$ (27\%) de los sedimentos. De sus 91 deslizamientos, 19 están a lo largo del sistema de arroyos que afectan el $11 \%$ de su área. A pesar de que el área y el número de deslizamientos en la rampa piroclástica indiferenciada es $\sim 4$ veces más grande que la de las laderas plegadas (calizas-dolomitas del Cretácico), esta forma del relieve sedimentaria se compara con la forma del relieve volcánico en cuanto al número de deslizamientos por kilómetro cuadrado y es la que contribuye con el mayor volumen de materiales por kilómetro cuadrado $\left(37545 \mathrm{~m}^{3} / \mathrm{km}^{2}\right)$. En el área de estudio, los valles fluviales están contenidos en 13 formas del relieve y cubren el $14 \%$ de la cuenca, si se tomaran en cuenta para el análisis de deslizamientos, esta forma del relieve abarcaría $\sim 58 \%$ de los deslizamientos, los cuales contribuyen con casi la mitad del volumen de materiales.

A pesar de no tener la misma proporción de deslizamientos, el volumen de materiales aportados para otras dos formas del relieve volcánicas (rampa piroclástica y flujos de lava (Holoceno) también es alto.

Las formas del relieve volcánico cubren $54 \mathrm{~km}^{2}$, de los cuales el $62 \%$ registra un volumen alto de materiales removilizados, el $27 \%$ registra un volumen de materiales moderado y el $11 \%$ reporta un volumen bajo. Los $51 \mathrm{~km}^{2}$ restantes del área de la cuenca están cubiertos por formas del relieve sedimentarias; de esta área, el $60 \%$ tiene un alto volumen de aporte de materiales y el $40 \%$ restante tiene un bajo volumen.

\section{DISCUSIÓN Y CONCLUSIONES}

El objetivo de este estudio fue modelar y evaluar el volumen de materiales aportados por deslizamientos en términos de formas del relieve. Sin embargo, el volumen de material es difícil de modelar debido a los cambios continuos en la topografía a causa de los deslizamientos posteriores, y factores antrópico-ambientales (cambio de uso del suelo, reforestación, erosión lateral y vertical de los ríos, etc.) en grandes áreas y de difícil acceso. El modelado y cálculo de volumen no es ideal con un MDT de baja resolución por el tamaño del pixel y/o con un MDS de alta resolución sin procesar creado a partir de un VANT debido al ruido diferente a la topografía en los modelos.

En este estudio, las mediciones geométricas de deslizamientos representativos se determinan mediante el uso de un VANT y un método que permitió obtener un MDT, de resolución moderada, de la topografía actual y previa a un deslizamiento a partir del cual se estimó el volumen aportado. Estas mediciones permitieron establecer una relación empírica área-volumen, expresada en forma de ley de potencias para calcular el volumen de deslizamientos de todo el inventario y por formas del relieve. El estudio muestra que el uso de un MDT de baja resolución como referencia para definir la topografía inicial del MDS del VANT, no está libre de errores en la eliminación del ruido (árboles, arbustos, etc.) y, por consiguiente, afectará la creación del MDT de resolución moderada y el cálculo del volumen. Cuando se usa un MDT de baja resolución, se necesita compensar estos errores de manera heurística (uso de pesos y filtros). La evaluación subjetiva de las condiciones actuales y previas de un deslizamiento en campo, basada en la experiencia del investigador, influirá en la delimitación del área de deslizamientos y el cálculo del volumen. En esta investigación se aplica una compensación para obtener un error vertical máximo de $\sim 1$ m en el MDT de moderada resolución mediante el uso heurístico de histogramas, ponderaciones y filtrado. Lo anterior implica una propagación potencial de error en la estimación del volumen que va de 0 a $0.25 \mathrm{~m}^{3}$ en cada pixel. Aunado a lo anterior, la topografía previa al deslizamiento se reconstruyó mediante un proceso de interpolación de valores altimétricos aledaños al deslizamiento. Por lo que, al no contarse con valores altimétricos internos de la superficie previa al deslizamiento, su reconstrucción no será precisa y variará con el tipo de interpolación usado. Otra posible fuente de error es el desplazamiento topográfico horizontal que puede existir si no hay una georreferenciación adecuada de las superficies actual y previa. En nuestro caso, esta fuente de error se eliminó, ya que la topografía previa al deslizamiento se reconstruyó a partir de la topografía actual expresada por el MDT de resolución moderada, manteniéndose así los mismos atributos espaciales para ambas superficies. Además, consideramos que el MDT de INEGI es correcto tanto en lo horizontal como en lo vertical y, al 
Tabla 1. Características de las formas del relieve y sus volúmenes calculados.

\begin{tabular}{|c|c|c|c|c|c|c|}
\hline Forma del terreno & $\begin{array}{c}\text { Área de la } \\
\text { forma del } \\
\text { terreno }\left(\mathrm{km}^{2}\right)\end{array}$ & $\begin{array}{l}\text { No. de deslizamientos } \\
\text { en la forma } \\
\text { del terreno }\end{array}$ & $\begin{array}{c}\begin{array}{c}\text { Área de } \\
\text { deslizamientos } \\
\left(\mathrm{m}^{2}\right)\end{array}\end{array}$ & $\begin{array}{l}\text { Volumen de material } \\
\text { en la forma del } \\
\text { terreno }\left(\mathrm{m}^{3}\right)\end{array}$ & $\begin{array}{c}\text { Densidad de } \\
\text { deslizamientos } \\
\left(\text { por } \mathrm{km}^{2}\right)\end{array}$ & $\begin{array}{l}\text { Densidad de } \\
\text { volumen } \\
\left(\mathrm{m}^{3} / \mathrm{km}^{2}\right)\end{array}$ \\
\hline $\begin{array}{l}\text { Relicto de colapso } \\
\text { volcánico }\end{array}$ & 0.4 & 0 & 0 & 0 & 0 & 0 \\
\hline $\begin{array}{l}\text { Coladas históricas de lava } \\
\text { (años } 1545,1566 \text { y 1613) }\end{array}$ & 1.5 & 0 & 0 & 0 & 0 & 0 \\
\hline $\begin{array}{l}\text { Coladas de lava (Pleistoceno } \\
\text { superior) }\end{array}$ & 4.7 & 13 & 21538 & 44150 & 2.8 & 9434 \\
\hline $\begin{array}{l}\text { Coladas de lava de Sierra Negra } \\
\text { (Pleistoceno) }\end{array}$ & 1.8 & 2 & 3379 & 6186 & 1.1 & 3488 \\
\hline $\begin{array}{l}\text { Laderas cubiertas de depósitos } \\
\text { piroclásticos }\end{array}$ & 4.0 & 0 & 0 & 0 & 0 & 0 \\
\hline Rampa piroclástica & 3.3 & 15 & 18725 & 34418 & 4.6 & 10513 \\
\hline Rampa piroclástica indiferenciada & 25.8 & 356 & 389303 & 829719 & 13.8 & 32146 \\
\hline $\begin{array}{l}\text { Lomeríos plegados } \\
\text { (caliza-dolomita del Cretácico) }\end{array}$ & 11.2 & 91 & 192001 & 420508 & 8.1 & 37587 \\
\hline Piedemonte $3-6^{\circ}$ & 6.7 & 0 & 0 & 0 & 0 & 0 \\
\hline Piedemonte $<3^{\circ}$ & 10.1 & 0 & 0 & 0 & 0 & 0 \\
\hline Rampa fluvioglacial & 3.6 & 0 & 0 & 0 & 0 & 0 \\
\hline Total & 105.4 & 607 & 738053 & 1547657 & 39 & 106349 \\
\hline
\end{tabular}

Tabla 2. Características geomorfológicas de las formas del relieve y sus volúmenes aportados en los valles.

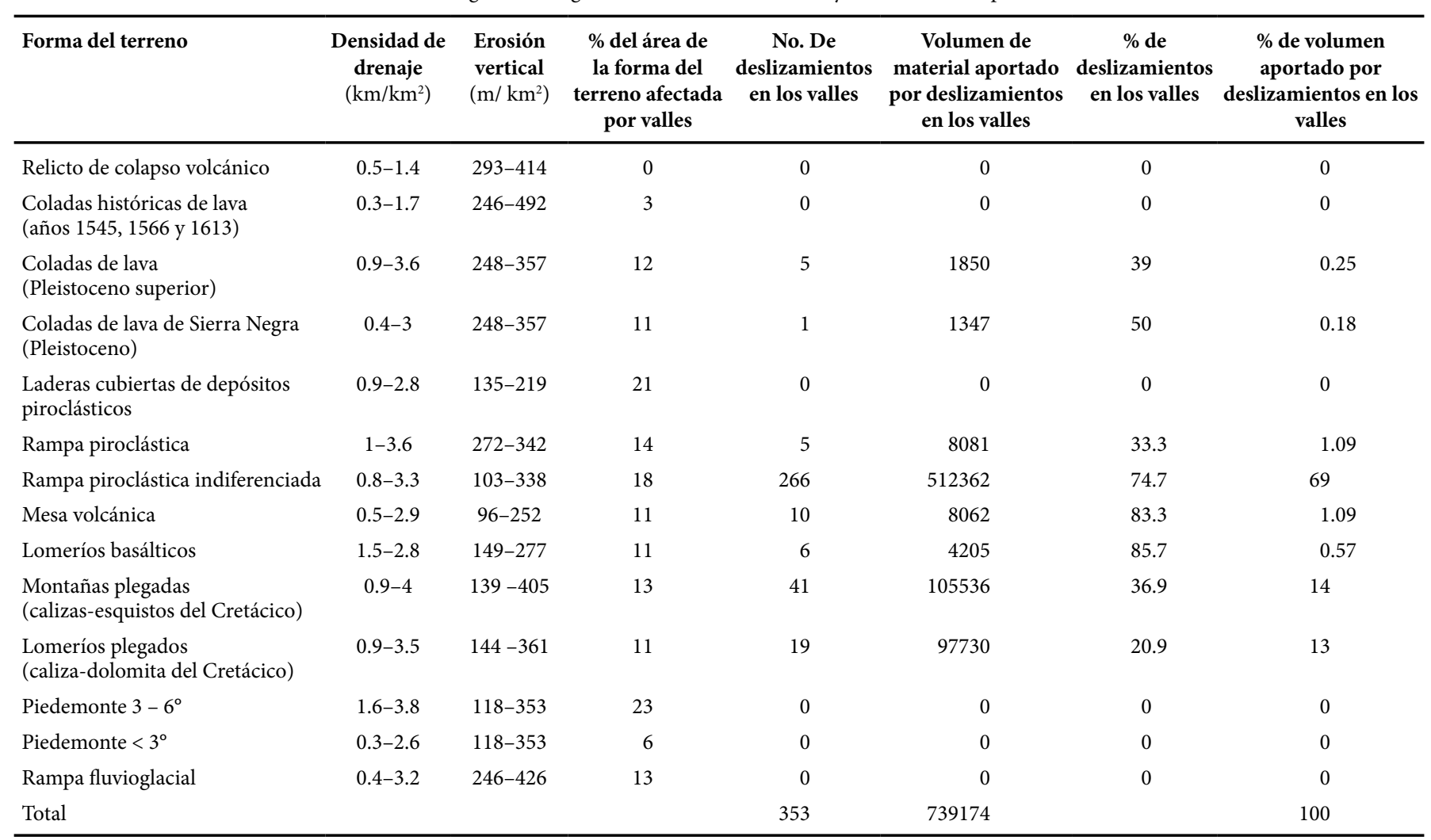




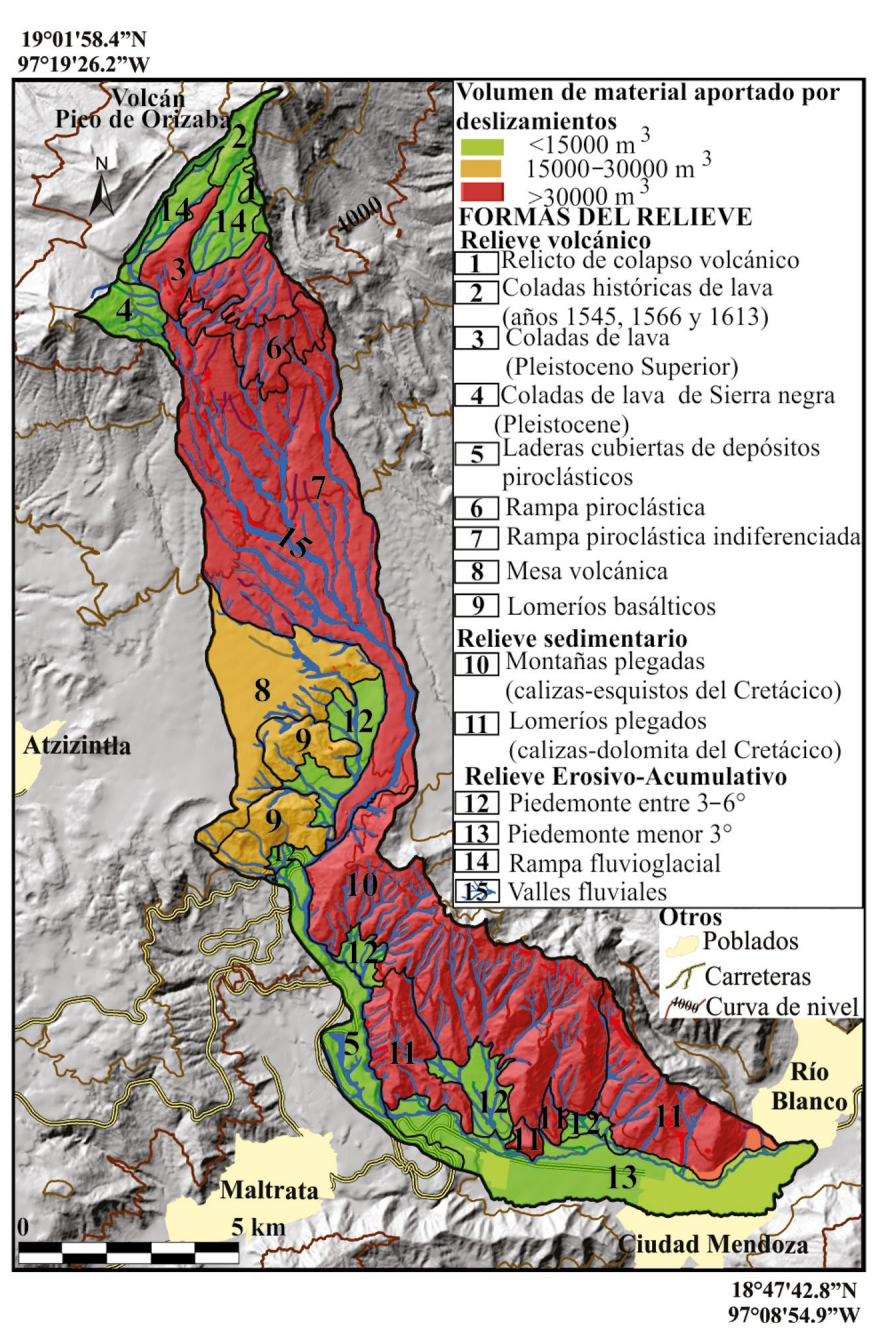

Figura 7. Aporte de volúmenes de materiales por formas del relieve.

usar el punto de anclaje del GPS para situar las imágenes del dron se redujo el error horizontal. No así el vertical, debido a la dificultad de acceso que limitó el uso más extensivo de puntos de control con GPS.

Los resultados muestran parcialmente que el uso del MDT de moderada resolución tiende a aumentar el volumen calculado. Así, por ejemplo, usando un deslizamiento de asentamiento profundo de $1738 \mathrm{~m}^{2}$, la diferencia entre el volumen calculado a partir del MDT de moderada resolución fue de aproximadamente 3.5 veces mayor que el calculado usando el MDS de alta resolución.

A pesar de los posibles errores introducidos por el procesamiento para generar la topografía de los deslizamientos y calcular su volumen, consideramos que la metodología provee una ruta viable y promisoria para monitorear, a bajo costo y en poco tiempo, grandes deslizamientos de difícil acceso. Así, por ejemplo, mediciones hechas con cinta métrica, estadal y GPS diferencial, requieren de un trabajo de campo intenso y de recursos, y no garantizan, a pesar de la exactitud de equipo, la correcta representación de la topografía. En particular, en el trabajo de Legorreta-Paulín et al. 2017, la medición de un sólo deslizamiento de $1744 \mathrm{~m}^{2}$ requirió de un equipo de seis personas y tres sesiones de dos días de trabajo. En estas mediciones los puntos de muestreo altimétrico se realizaron por medio de transectos, usando GPS diferencial y rapel por lo escarpado del terreno. Esto implicó puntos de muestreos no equidistantes para generar la topografía. En este trabajo, tales inconvenientes fueron eliminados mediante el uso del VANT, pero también se introdujeron, como ya se ha mencionado, nuevos elementos potenciales de error (de posicionamiento vertical y estimación de volumen).

Los datos morfométricos de los deslizamientos seleccionados permitieron establecer una relación teórica que explica hasta un $71 \%$ el volumen de los deslizamientos con base en su área. El grado de ajuste de esta relación teórica tal vez pueda ser mejorado incrementando el número de muestreo, ya que algunos de los deslizamientos mostraron una mayor dispersión. Tal dispersión podría deberse a las condicionas geológicas que propician que algunos deslizamientos tengan una mayor incisión vertical y, por consiguiente, de volumen aportado. Es necesario que futuros estudios teóricos se realicen para definir con más claridad las ventajas y desventajas de estos métodos en el cálculo de volumen.

Los resultados también indican que las formas del relieve que representan condiciones geológicas, geomorfológicas y fisiográficas homogéneas son útiles en la cuantificación, evaluación y modelado de la producción de sedimentos de deslizamientos. Por ejemplo, la rampa piroclástica indiferenciada y la de lomeríos plegados (calizadolomita del Cretácico) tuvieron el mayor volumen de materiales por kilómetro cuadrado. Ambas formas del relieve tienen una alta densidad de valles y erosión vertical, pero las condiciones geológicas hacen una diferencia. Si bien la unidad volcánica tiene el mayor número de deslizamientos por kilómetro (14) y es la unidad de relieve más grande en la cuenca $\left(26 \mathrm{~km}^{2}\right)$, no es la primera unidad en la contribución de sedimentos $\left(32159 \mathrm{~m}^{3} / \mathrm{km}^{2}\right)$. La forma del relieve sedimentaria tiene la mayor producción de sedimentos por kilómetro cuadrado (37545 $\mathrm{m}^{3} / \mathrm{km}^{2}$ ). Estas diferencias se deben a que las calizas y dolomitas que la conforman han estado expuestas a períodos más prolongados al intemperismo, en comparación con la unidad volcánica. Además, el plegamiento, el buzamiento y la presencia de fallas y fracturas de las rocas sedimentarias contribuye al desplazamiento de fragmentos y sedimentos por procesos gravitacionales. Asimismo, la mayoría de los deslizamientos están ubicados a lo largo del valle $(75 \%)$ en la forma de relieve volcánica, mientras que en la forma de relieve sedimentaria de Lomeríos plegados (caliza-dolomita del Cretácico) el porcentaje es de sólo $21 \%$. Esto se debe a que el material suelto que conforma la unidad de la rampa piroclástica indiferenciada permite una erosión vertical más activa a lo largo de las paredes del río, que a su vez aumenta las pendientes pronunciadas que provocan una gran cantidad de deslizamientos a lo largo del valle. En este trabajo se estima un aproximado de $638 \mathrm{~m}^{3} / \mathrm{km}^{2} /$ año de sedimentos aportados en la cuenca, sin embargo, consideramos que este valor subestima la magnitud real debido a que muchos de los deslizamientos en el área de estudio ya habían ocurrido previamente a la elaboración del inventario y, por consiguiente, se desconoce el área y el volumen original desplazado. Es necesario la elaboración a futuro de un nuevo inventario y estrategias que permitan comparar y dar seguimiento a deslizamientos ya catalogados para calcular la magnitud de sedimentos.

A pesar de los desafíos en esta metodología, el modelado y cálculo del volumen de materiales de deslizamientos proporciona un paso importante para la planificación de la gestión de desastres en áreas en las que no hay información topográfica de alta resolución y donde el acceso para realizar mediciones con cinta, estadal o GPS diferencial se ve limitado por lo accidentado del terreno.

\section{REFERENCIAS}

Alanis-Anaya, R.M., 2017, Influencia del cambio de uso de suelo en la inestabilidad de la ladera en la subcuenca del Río Chiquito-Barranca del Muerto, volcán Pico de Orizaba: Ciudad de México, México, Universidad Nacional Autónoma de México, tesis de Doctorado en Geografía, 221 pp. 
Broeckx, J., Vanmaercke, M., Bălteanu, D., Chendeș, V., Sima, M., Enciu, P., Poesen, J., 2016, Linking landslide susceptibility to sediment yield at regional scale: application to Romania: Geomorphology, (268), 222-232.

Capra, L., Coviello, V., Borselli, L., Márquez-Ramírez, V. H., ArámbulaMendoza, R., 2018, Hydrological control of large hurricane-induced lahars: evidence from rainfall-runoff modeling, seismic and video monitoring: Natural Hazards and Earth System Sciences, 18(3), 1-36.

CENAPRED (Centro Nacional de Prevención de Desastres), 2017, Elaboración de escenarios de peligro por inundación y deslizamiento de laderas, mediante información obtenida por Vehículos aéreos no tripulados-drones (VANT). Informe trimestral del ejercicio de recursos y el informe técnico del avance de proyecto. H00-DG/0540/017: Ciudad de México, México, Secretaría de Gobernación, Coordinación Nacional de Protección Civil, Sistema de Protección Civil , 13 pp.

Colomina, I., Molina, P., 2014, Unmanned aerial systems for photogrammetry and remote sensing: A review: ISPRS journal of photogrammetry and remote sensing, 92, 79-97.

CFE (Comisión Federal de Electricidad), 2006, Proyecto para el control integral de torrentes en la cuenca del Río Blanco, Veracruz, México en IV Foro mundial del agua: Ciudad de México, México, Comisión Federal de Electricidad, 38 pp.

Díaz, S.R., Cadena, E., Adame, S., Dávila, N., 2019, Landslides in Mexico: theiroccurrence and social impactsince 1935: Landslides, 1-16.

Edmonds, R., 2017, High resolution mapping, description and interpretation of the foreshore in front of the great under cliff landslides; the Plateau, Dowlands and Bindon within the Axmouth to Lyme Regis Under cliffs National Nature Reserve, south Devon coast, England, and how that might inform understanding of the mechanisms of failure, in Proceedings of the Geologists' Association: England 130 (3-4), 473-482.

Ferrari, L., Orozco-Esquivel, T., Manea, V., Manea, M., 2012, The dynamic history of the Trans-Mexican Volcanic Belt and the Mexico subduction zone: Tectonophysics, (522), 122-149.

Francioni, M., Coggan, J., Eyre, M., Stead, D., 2018, A combinedfield/ remotesensingapproachforcharacterizinglandsliderisk in coastal áreas: International Journal of Applied Earth Observation and Geoinformation, 67, 79-95.

García, E., 2004, Modificaciones al sistema de clasificación climática de Köppen: para adaptarlo a las condiciones de la República Mexicana, 5ta ed., México, Instituto de Geografía, Universidad Nacional Autónoma de México, 90 pp.

Guzzetti, F., Reichenbach, P., Cardinali, M., Galli, M., Ardizzone, F., 2005, Probabilistic landslide hazard assessment at the basin scale: Geomorphology ,(72), 272-299.

Guzzetti, F., Ardizzone, F., Cardinali, M., Rossi, M., 2009, Landslide volumes and landslide mobilization rates in Umbria, central Italy: Earth and Planetary Science letters, 279, 22-229.

Hernandez A.B., Ruiz, R.N., 2016, The production of vulnerability to landslides: the risk habitus in two landslide-prone neighborhoods in Teziutlán, Mexico: Investigaciones Geográficas, 90, 7-27.

INEGI (Instituto Nacional de Estadística, Geografía e Informática), 2015 Carta topográfica Puebla-Veracruz de Ignacio de la Llave e14b56a, Escala 1:20,000: Aguascalientes, México, Instituto Nacional de Estadística, Geografía e Informática,, 1 mapa.

Iwahashi, J., Watanabe, S., Furuya, T., 2001, Landform analysis of slope movements using DEM in Higashikubiki area, Japan: Computer \& Geosciences, 27(7), 851-865.

Jauregui, E., 1995, Rainfall fluctuations and tropical storm activity in Mexico: Erdkunde, 49, 39-48.

Kaldaron-Asael, B., Katz, O., Aharonov, E., Marco, S., 2008, Modeling the relation between area and volume of landslides: Jerusalem, Israel, Ministry of National Infrastructures Geological Survey of Israel,, Report for Steering Comittee for Earthquakes, 16 pp.

Legorreta-Paulín, G., Bursik, M., Pouget, S., Serdar, C., Hubp, J.L., 2014 Mapping landforms for landslide hazards assessment on the SW flank of Pico de Orizaba volcano, Puebla-Veracruz, Mexico: Zeitschriftfür Geomorphologie, 58(1), 81-93.

Legorreta-Paulín, G., Bursik, M., Zamorano-Orozco, J.J., Lugo-Hubp, J., Martinez-Hackert, B., Bajo Sánchez, J.V., 2017, Estimación del volumen de los depósitos asociados a deslizamientos a través de geoformas, en el flanco SW del volcán Pico de Orizaba, Puebla-Veracruz: Investigaciones
Geográficas, 92, 21-33.

Lugo-Hubp, J., Córdova, C., 1992, Regionalización geomorfológica de la República Mexicana: Investigaciones Geográficas, 25, 25-63.

Lugo-Hubp, J., Zamorano-Orozco, J.J., Capra, L., Inbar, M., Alcántara-Ayala, I., 2005, Los procesos de remoción en masa en la Sierra Norte de Puebla, octubre de 1999: causa y efectos: Revista Mexicana de Ciencias Geológicas, 22(2), 212-228

Muñoz-Salinas, E., Renschler, C.S., Palacios, D., 2009, A GIS-based method to determine the volume of lahars: Popocatepetl volcano, Mexico: Geomorphology, 111 (1-2), 61-69.

Oliva-González, A.O., Jiménez, D.M., Alvarez-Garcia, I.N., Nicieza, C.G., Álvarez-Vigil, A.E., 2014, Hill side instability in the Tijuana metropolitan area. Analysis of landslide-provoked building collapse: Engineering Failure Analysis, 46, 166-178.

Parrot, J.-F., 2011, Dem_lidar_generation, Módulo ejecutable MS-DOS: Universidad Nacional Autónoma de México, México, no publicado.

Parrot, J.-F., 2012, Software DEMONIO (Digital Elevation Models Obtained by Numerical Interpolating Operations): Universidad Nacional Autónoma de México, México, Copyright Instituto Nacional de Derecho de Autor (INDAUTOR): 03-2012-120612205000-01.

Parrot, J.-F., 2016, Generación de MDE a partir de datos vectoriales. Paquete de Módulos Ejecutables desarrollados en $\mathrm{C}++$ : Universidad Nacional Autónoma de México, México, Copyright Instituto Nacional de Derecho de Autor (INDAUTOR): 03-2016-103110072200-01.

Parrot, J.F., 2018a, Software Select Slices: Universidad Nacional Autónoma de México, México, Copyright Instituto Nacional de Derecho de Autor (INDAUTOR): 03-2018-011109492800-01.

Parrot, J.F., 2018b, Shaving, V 1.0, Módulo ejecutable MS-DOS: Universidad Nacional Autónoma de México, México, no publicado.

Ouédraogo, M. M., Degré, A., Debouche, C., Lisein, J., 2014, The evaluation of unmanned aerial system-based photogrammetry and terrestrial laser scanning to generate DEMs of agricultural watersheds: Geomorphology, $214,339-355$.

Rodríguez, S.R., Mora-González, I., Murrieta-Hernández, J.L., 2006, Flujos de baja concentración asociados con lluvias de intensidad extraordinaria en el flanco sur del volcán Pico de Orizaba (Citlaltépetl), México: Boletín de la Sociedad Geológica Mexicana, 58(2), 223-236.

Rodríguez, S., Mora, I., Murrieta, J., Morales, B.W.V., 2011, Peligros geológicos más frecuentes en el estado de Veracruz: Veracruz, México, Universidad Veracruzana Xalapa, https://ebooks.uv.mx/pdfreader/peligros-geolgicosms-frecuentes-en-el-estado-de-veracruz, 85 pp., consulta: 15 de marzo de 2020.

Ruiz, C.R., Corominas, D.J., Hurlimann, Z.M., 2017, Experiencias con drones para el estudio de movimientos de ladera, en IX Simposio Nacional sobre Taludes y Laderas Inestables: Barcelona, España. Universidad Politécnica de Catalunya, https://upcommons.upc.edu/bitstream/handle/2117/107109/ Experiencias\%20con\%20drones\%20para\%20el\%20estudio\%20de\%20 movimientos\%20de $\% 20$ laderas.pdf? sequence $=1$ \&isAllowed $=y$, consulta: 15 de marzo de 2020.

Šiljeg, A., Barada, M., Marić, I., Roland V., 2019, The effect of user defined parameters on DTM accuracy - development of hybrid model: Applied Geomatics, 11(1), 81-96.

Washington State Department of Natural Resources, Forest Practices Division (WS-DNR:FPD), 2006, Landslide Hazard Zonation (LHZ) Mapping Protocol, version 2.0, http://www.dnr.wa.gov/BusinessPermits/Topics/ LandslideHazardZonation/Pages/fp_lhz_review.aspx, consulta: 25 de octubre de 2019.

Wenkey, D., Jen, C.H., Böse, M., Lin, J.C., 2012, Assessment of sediment delivery from successive erosion on stream-coupled hill slopes via a time series of topographic surveys in the central high mountain range of Taiwan: Quaternary International, 263, 14-25. Doi: 10.1016/j.quaint.2011.02.018.

Manuscrito recibido: agosto 11, 2019

Manuscrito corregido recibido: enero 13, 2020

Manuscrito aceptado: enero 20, 2020 